\title{
Preferencia de metas relacionadas con ejercicio físico en mujeres con fibromialgia. ¿Hacer ejercicio o evitar el dolor?
}

\section{Preference for goals related to physical exercise in women with fibromyalgia. Exercise or avoid pain?}

Fecha de recepción: 10-06-2019

Fecha de aceptación: 15-11-2019
Eva León Zarceño

Universidad Miguel Hernández de Elche

Fermín Martínez-Zaragoza

Universidad Miguel Hernández de Elche

Sofía López-Roig Universidad Miguel Hernández de Elche

Cecilia Peñacoba

Universidad Rey Juan Carlos

María Ángeles Pastor-Mira

Universidad Miguel Hernández de Elche

\section{resumen/alsstract:}

Introducción: La fibromialgia es una enfermedad crónica con dolor musculo-esquelético como síntoma principal. El ejercicio físico es una herramienta óptima para la mejora de los síntomas. Las pacientes asocian empeoramientos a la ejecución de actividad física, por lo que acaban por evitarla. Los objetivos fueron: (1) Evaluar y describir la preferencia por metas de logro relacionadas con ejercicio físico frente a metas de evitación del dolor y su perfil sociodemográfico y clínico y (2) establecer la asociación entre esa preferencia de metas y diferentes patrones de actividad física. Método: se utilizó un diseño correlacional y transversal, evaluando a 260 mujeres con fibromialgia en preferencia de metas respecto a ejercicio físico, patrones de actividad y estado emocional. Resultados: Las mujeres con preferencia por metas de ejercicio físico no puntuaron significativamente diferente a las mujeres que mostraron la preferencia contraria en ninguna de las variables sociodemográficas y clínicas estudiadas. La preferencia por evitar el dolor se asoció significativamente a patrones de actividad física de evitación y de menor persistencia. Conclusiones: Las pacientes suelen preferir metas relacionadas con la evitación del dolor. Esta preferencia no se asocia a ningún perfil sociodemográfico o clínico, pero sí a diferentes patrones de actividad. Dada la relevancia del ejercicio físico en el tratamiento, intervenir para que sea una meta relevante se configura como una meta terapéutica.

Introduction: Fibromyalgia is a chronic disease with musculoskeletal pain as main symptom. Physical exercise is an optimal strategy for the improvement of the symptoms. Patients associate deteriorate physical activity, so they end up avoiding it. The objectives were: (1) Evaluate and describe the preference for achievement goals related to physical exercise versus avoidance goals pain and sociodemographic and clinical profile, and (2) establish the association between that setting goals and different patterns of physical activity. Method: A correlational design was used, evaluating 260 women with fibromyalgia in preference to goals regarding physical exercise, activity patterns and emotional state. Results: Women with preferences for physical exercise goals did not score significantly differently than women who showed the opposite preference in the sociodemographic and clinical variables studied. The preference to avoid pain was significantly associated with patterns of avoidance of physical activity and less persistence. Conclusions: Patients generally prefer goals related to pain avoidance. This preference is not associated with any sociodemographic or clinical profile, but with different activity patterns. Given the relevance of physical exercise in the treatment, intervening to be a relevant goal is configured as a therapeutic goal. 


\section{palabras clave/keywords:}

Fibromialgia, dolor crónico, metas, ejercicio físico, mujer

Fibromyalgia, chronic pain, goals, physical exercise, woman

\section{Introducción}

La enfermedad crónica ha sido definida por la Organización Mundial de la Salud (OMS, 2019) como aquella enfermedad que presenta una progresión lenta y de larga duración. En nuestra sociedad estas enfermedades han alcanzado una intensa atención sanitaria destinando recursos a su tratamiento y suponiendo elevados costes no solo económicos, sino sociales y psicológicos (León y Boix, 2018). La fibromialgia, concretamente, es una enfermedad crónica caracterizada por dolor crónico musculo-esquelético generalizado y difuso. En el curso de la enfermedad suelen presentarse otros síntomas como la fatiga, problemas de sueño, ansiedad, depresión y dificultades cognitivas, entre otros. La investigación y los diferentes documentos de consenso, como el publicado por EULAR, ha considerado la práctica de ejercicio en esta enfermedad como la primera opción de intervención en el marco de una estrategia terapéutica escalonada (Macfarlane et al., 2017). Existen evidencias de los efectos positivos del ejercicio en los resultados de salud cuando se incluye como parte del tratamiento (Busch et al., 2013). Los expertos indican que los ejercicios combinados de fuerza y flexibilidad son recomendables y que la prescripción debería comenzar a baja intensidad y de corta duración incrementándose gradualmente (Sañudo, Galiano, Carrasco, y de Hoyo, 2010). Sin embargo, la evidencia científica indica que las mujeres con fibromialgia son menos activas que otras mujeres con características socio-demográficas semejantes (McLoughlin, Colbert, Stegner, y Cook, 2011) o realizan con poca frecuencia ejercicio físico regular como puede ser andar (López-Roig et al., 2016).

Este panorama muestra una realidad: a medio y largo plazo, el ejercicio es beneficioso para las mujeres con fibromialgia; sin embargo, estas mujeres presentan serias dificultades para realizarlo. En este sentido, las personas con problemas de dolor pueden anticipar empeoramientos asociados a la actividad y, por tanto, evitarla (Cristhiansen, Oettingen, Dahme, y Klinger, 2010) lo que supone que se promueva la inactividad (Karsdorp, Geenen, Kroese y Vlaeyen, 2016). Diferentes modelos relacionan este hecho con cuestiones motivacionales y de miedo al dolor/movimiento (Vlaeyen y Linton, 2012). Actualmente, los modelos motivacionales sobre dolor crónico explican el comportamiento de las personas que lo padecen sobre la base de múltiples metas que pueden competir entre sí en una misma situación (Karsdorp y Vlaeyen, 2011). Así, ante la práctica del ejercicio físico, las personas con dolor podrían optar por metas evitadoras o hedónicas (preferirían evitar el dolor y no iniciar o detener el ejercicio) o por metas de logro a largo plazo (preferirían iniciar o mantenerse en la actividad). El estudio de la actividad física en estas pacientes ha destacado la existencia de diferentes patrones en dolor crónico relacionados tanto con la persistencia en continuar con la tarea, a pesar del dolor como con la evitación de la actividad física (Kindermans, Roelofs, Goossens, Huijnen, Verbunt, y Vlaeyen, 2011). Este trabajo se encuadra desde la 
perspectiva motivacional del modelo de evitación del dolor (Van Damme y Kindermans, 2015) y de los procesos de autorregulación en el establecimiento y logro de metas de Heckhausen y Gollwitzer (1987).

La psicología de la actividad física y el deporte, como campo de actuación profesional, tiene entre sus ámbitos de actuación el estudio de los factores y los efectos psicológicos implicados en la práctica de la actividad física de poblaciones especiales, así como el uso del ejercicio como parte de tratamientos de mejora de problemas de salud (Cantón, 2009). En este contexto, el trabajo con diferentes especialistas implicados en el ámbito de la salud es de suma importancia para obtener los máximos beneficios de la práctica de ejercicio en la población a la que se dirige la intervención (León y Cantón, 2008). En este sentido, el psicólogo del deporte debe tener en cuenta en el diseño de cada intervención psicológica, entre otros aspectos, las repercusiones físicas y psicológicas de la enfermedad crónica en el paciente (León y Boix, 2018). Conocer si las metas de las personas con fibromialgia favorecen la realización de ejercicio físico y si esas metas apoyan o no patrones de actividad relacionados con la mejora de resultados de salud puede aportar evidencia para diferentes tipos de intervención adaptadas a sus características diferenciales.

Por ello, los objetivos de este trabajo fueron: (1) Evaluar y describir la preferencia por metas de logro relacionadas con ejercicio físico frente a metas de evitación del dolor y su perfil sociodemográfico y clínico y (2) establecer la asociación entre esa preferencia de metas y diferentes patrones de actividad física.

Respecto a las hipótesis se espera que existirá un perfil sociodemográfico y clínico de mujeres con fibromialgia diferente en aquellas mujeres que prefieran metas de logro o las que prefieren metas evitadoras y esto, a su vez, tendrá relaciones con el patrón de actividad física que muestran.

\section{Método}

\section{Diseño}

El estudio sigue un diseño descriptivo, correlacional y de tipo transversal.

\section{Participantes}

La muestra del estudio estuvo compuesta por 260 mujeres con diagnóstico de fibromialgia. Un $62.7 \%$ ( $n=163$ ) eran pacientes atendidas en la Unidad de Fibromialgia del Hospital de San Vicente del Raspeig (Alicante) y un 37.3\% (n=97) mujeres de diferentes asociaciones de la Comunidad Valenciana. La edad media fue de $51.2(d t=8.7)$. Un $71.2 \%$ de ellas estaba casada y un $36.9 \%$ tenía estudios primarios, mientras que el $38.5 \%$ tenía estudios secundarios. Del total de participantes un $68.4 \%(n=178)$ no trabajaba mientras que el resto permanecían laboralmente activas. Un $87 \%$ de las pacientes se ocupa del trabajo de casa mientras que el $59.10 \%$ tiene ayuda para el trabajo de casa. El tiempo medio desde la aparición de los primeros síntomas de la fibromialgia fue de 15.9 años $(d t=7.9)$, y desde el diagnóstico 7.9 años $(d t=8.0)$.

Los criterios de inclusión para participar en el estudio fueron: mujeres, entre 18-70 años, con diagnóstico de fibromialgia, nivel educativo que permita la autoadministración de cues- 
tionarios y con capacidad comprensiva. Se excluyeron pacientes con comorbilidad que impidiese la práctica de ejercicio físico.

\section{Instrumentos}

Se administró un cuestionario sociodemográfico y clínico elaborado ad hoc para registrar datos clínicos y sociodemográficos, tales como la edad o los años de curso de enfermedad.

Para evaluar la preferencia de metas sobre ejercicio físico en pacientes con fibromialgia, se utilizó el cuestionario GPQ-E (GPQ-Ejercicio). El dato de la consistencia interna del cuestionario se encuentra en el apartado de los resultados. Este cuestionario es una adaptación del Goal Pursuit Questionnaire (GPQ) de Karsdorp y Vlaejen (2011), que evalúa preferencia de metas en dolor crónico. EI GPQ-E consta de cinco ítems. Los ítems se seleccionaron comparando y adaptando los de los cuestionarios IPAQ-S (Craig et al., 2003), LTPAI (Munguía-Izquierdo, Legaz-Arrese, y Mannerkorpi, 2011) y de la escala de autoeficacia para la actividad física (Fernández- Cabrera, Medina- Anzano, Herrera-Sánchez, Rueda-Méndez, y Fernández Del Olmo, 2011). Se redactaron siguiendo la estructura del cuestionario original pero cambiando la especificación de dolor localizado (dolor en el brazo, muñeca, espalda, etc.) por dolor generalizado, típico en la fibromialgia. En cada ítem se plantea a la paciente una situación relacionada con el ejercicio físico (que implica actividad desde ligera a intensa). En el desarrollo de esa situación se plantea el aumento del dolor y la importancia de finalizar la actividad, pidiéndole que piense cuál sería su preferencia: mantenerse en la actividad (meta de logro) o evitar el dolor que pueda sentir en ese momento (meta hedónica). Se contesta mediante una escala de respuesta tipo Likert de 6 puntos $(1=$ totalmente en desacuerdo, 6= totalmente de acuerdo), donde las puntuaciones más altas indican una mayor preferencia por evitar el dolor. La puntuación total se obtiene calculando la media de todos ítems. Cuanto más alta es la puntuación, mayor preferencia por evitar el dolor. En esta muestra, se obtuvo un $=.93$.

El estado emocional se evaluó en términos de ansiedad y depresión con la Escala de Ansiedad y Depresión Hospitalaria (versión española de Terol et al., 2007). Este cuestionario está compuesto por 14 ítems, 7 para cada subescala, con un formato de respuesta tipo Likert de 4 puntos con diferentes anclajes verbales en función del ítem. Cuánto más elevada sean las puntuaciones en las respectivas subescalas, mayores niveles de ansiedad y depresión. La consistencia interna en población española en un estudio con pacientes de fibromialgia ha sido estimada con un $=.83$ para ansiedad y con $=.87$ para depresión (Vallejo, Rivera, EsteveVives y Rodríguez-Muñoz, 2012). En esta muestra, para la escala de ansiedad, se obtuvo un $=.87$, y para la escala de depresión, $=.85$.

Los patrones de actividad en fibromialgia fueron evaluados a través de la adaptación española de la Escala de Patrones de Actividad (Esteve et al., 2016). Este instrumento se compone de 24 items contestados con una escala Likert de 5 puntos ( $0=$ Nunca, $4=$ Siempre) y agrupados en 8 subescalas que recogen los diferentes patrones de actividad: evitación del dolor ( = .75), evitación de actividad ( = .55), persistencia dependiente de la tarea ( = .84), persistencia excesiva ( = .65), persistencia dependiente del dolor $(=.78)$, regulación de la actividad para aumentar sus niveles $(=.73)$, regulación de la actividad para conservar 
energía para actividades valiosas $(=.81)$ y regulación de la actividad para reducir el dolor $(=.82)$. Entre paréntesis se describen los índices obtenidos en la muestra de estudio para cada subescala.

Finalmente, el impacto de la fibromialgia se evaluó con la adaptación española del FIQ-R (Salgueiro et al., 2013). Este cuestionario consta de 21 items y proporciona puntuaciones para función física $(=.81)$, impacto $(=.84)$ y síntomas $(=.76)$ de la fibromialgia, además de ofrecer una puntuación total indicando el impacto global (rango 0-100; = .83). A mayor puntuación, mayor impacto percibido.

\section{Procedimiento}

Este estudio es parte de otro estudio más amplio donde se ha valorado la preferencia de metas en personas con fibromialgia y su impacto en los resultados de salud en interacción con el afecto y la mediación de patrones de actividad de evitación y de persistencia. Se obtuvo la autorización de los comités éticos del Hospital General y Universitario de Alicante y de la Universidad Miguel Hernández de Elche. Posteriormente, tras la firma del consentimiento informado (y siguiendo las recomendaciones de la declaración de Helsinki para la investigación con humanos recogidas por la World Medical Association (WMA, 1964)) se evaluó a las pacientes. Se obtuvo el consentimiento informado de todas las participantes.

Una persona del equipo de investigación facilitaba los cuestionarios a las pacientes que, o bien lo realizaban en la consulta, tanto de manera individual como grupal, o, de forma excepcional, se lo llevaban y los traían cumplimentado en la siguiente cita médica. Además, se diseñó un cuestionario on-line para aquellas que podían hacerlo mediante esta vía.

\section{Análisis estadístico}

Se realizaron análisis descriptivos y de frecuencias de las variables sociodemográficas y médicas. Se realizó un análisis factorial exploratorio del GPQ-E utilizando el método de Máxima Verosimilitud (ML) y rotación oblicua. Se obtuvo el índice de Kaiser-Meyer-Olkin y la prueba de esfericidad de Bartlett para explorar el muestreo y la adecuación de los datos. Se seleccionaron los ítems con cargas mayores de .45 .

Aplicando el criterio de corte de puntuación media \pm 1 desviación típica en la variable preferencia de metas, se configuraron dos grupos: mujeres con preferencia de metas por evitar el dolor vs mujeres con metas de logro de ejercicio físico. Se estudiaron las diferencias en variables sociodemográficas y clínicas entre ambos grupos. Para la diferencia de medias para variables cuantitativas, se utilizaron pruebas $t$ para muestras independientes y para las variables categóricas, tablas de contingencia y el Test Exacto de Fisher. Para estudiar la asociación entre variables cuantitativas, se obtuvieron los coeficientes de correlación de Pearson entre la preferencia de metas y los patrones de actividad física. Se utilizó la prueba alfa de Cronbach para el análisis de la consistencia interna. El paquete estadístico utilizado fue el programa estadístico IBM SPSS Statistics 24 y el paquete estadístico $R$ (R Core Team, 2019). El nivel de significación fue marcado en .05. 


\section{Resultados}

El análisis factorial del GPQ-E mostró un único factor con un $74 \%$ de varianza explicada y en el que saturaron los cinco ítems (Tabla 1). El coeficiente de consistencia interna fue $=.93$. La puntuación media en el GPO-E fue 4.02 (DT = 1.5; rango 1-6). Aplicando el criterio menTabla 1.- Análisis de ítems y análisis factorial, descriptivos y consistencia interna del GPQ-E

\begin{tabular}{|c|c|c|c|c|c|c|c|}
\hline Item & GPQ-E Pienso que es más importante... & Carga factorial & $M D T$ & $A$ & C & $r_{1-T}$ & \\
\hline & Factor I. Metas de Evitación del dolor-ejercicio & & 4.01 .5 & -.5 & .7 & & .93 \\
\hline 1 & ... que disminuya ahora el dolor de mi cuerpo que terminar el ejercicio'. & .82 & 3.91 .6 & .3 & -1 & .87 & .90 \\
\hline 2 & ... que disminuya ahora el dolor de mi cuerpo que terminar el ejercicio². & .80 & 4.01 .6 & -4 & .9 & .87 & .90 \\
\hline 3 & $\begin{array}{l}\text {... que disminuya ahora el dolor de mi cuerpo que acabar de hacer este } \\
\text { ejercicio caminando. }\end{array}$ & .76 & 4.01 .7 & -.4 & -1 & .85 & .91 \\
\hline 4 & ... que disminuya ahora el dolor de mi cuerpo que terminar el ejercició. & .73 & 4.31 .6 & -6 & .8 & .84 & .91 \\
\hline 5 & ... que disminuya ahora el dolor de mi cuerpo que terminar el trayecto. ${ }^{5}$. & .47 & 3.91 .8 & -3 & -1.2 & .67 & .94 \\
\hline
\end{tabular}

$\mathrm{A}=$ Asimetría; $\mathrm{C}=$ Curtosis; $\mathrm{r}_{\mathrm{I}-\mathrm{T}}=$ Correlación ítem test; GPQ-E (GPQ-Exercise): ${ }^{\mathrm{a}}=$ los niveles de intensidad baja, moderada y alta se explican entre paréntesis en los ítems del cuestionario ${ }^{1}$ Actividad física suave, como dar un paseo.

${ }^{2}$ Actividad física moderada, como bailar.

${ }^{3}$ Actividad física moderada-intensa, como caminar rápido.

${ }^{4}$ Actividad física intensa, como correr.

${ }^{5}$ Actividad física ligera, como ir de compras.

cionado obtuvimos dos grupos: mujeres con preferencia de metas por el ejercicio físico $n=$ $34 ; M=1.37 ; D T=0.43$; rango 1-2) y mujeres con preferencia de metas por evitar el dolor ( $n$ $=87 ; M=5.54 ; D T=0.42$; rango $5-6$ ).

El perfil sociodemográfico y clínico de los dos grupos según la preferencia de metas por el ejercicio o por evitar el dolor es semejante (Tabla 2). No se obtuvieron diferencias significativas en las variables sociodemográficas y clínicas entre los dos grupos a excepción de la variable "se ocupa del trabajo de casa" (Fisher Exact Test $p=.002$ ), siendo de un $88 \%$ el porcentaje de mujeres con metas orientadas al ejercicio físico que realizan tareas de casa frente a las que no las realizan, y del $78 \%$ en el caso de las mujeres con metas evitativas. Sólo se hallaron tendencias (marginalmente significativas) relacionadas con la preferencia de metas en las variables edad $(t=-1.83, p=.06), y$ horas /día de trabajo en casa $(t=1.85, p$ $=.06)$.

En cuanto a las relaciones entre preferencia de metas y los patrones de actividad, se encontró que la preferencia de metas correlacionó significativamente con los diferentes pa- 
Tabla 2.- Diferencias entre grupos de preferencia de metas en variables sociodemográficas y clínicas

\begin{tabular}{|c|c|c|c|c|}
\hline & $\begin{array}{l}\text { Preferencia por el } \\
\text { ejercicio físico }^{\text {a }}\end{array}$ & $\begin{array}{l}\text { Preferencia por } \\
\text { evitar el dolor }{ }^{\mathrm{a}}\end{array}$ & $\mathrm{t}$ & $\mathrm{p}$ \\
\hline Edad & $48.7(9.2)$ & $52.0(9.2)$ & -1.83 & $.06^{+}$ \\
\hline Trabajo (horas/día) & $2.9(3.8)$ & $2.8(3.7)$ & 0.06 & .94 \\
\hline $\begin{array}{l}\text { Trabajo casa (horas/ } \\
\text { día) }\end{array}$ & $3.9(3)$ & $3.0(2.5)$ & 1.85 & $.06^{+}$ \\
\hline $\begin{array}{l}\text { Tiempo desde inicio } \\
\text { dolor (años) }\end{array}$ & $15.0(12.6)$ & $16.3(10.6)$ & -0.53 & .59 \\
\hline $\begin{array}{l}\text { Tiempo desde } \\
\text { diagnostico (años) }\end{array}$ & $6.1(5.6)$ & $8.2(7.9)$ & -1.27 & .20 \\
\hline Intensidad del dolor & $7.1(1.3)$ & $7.0(1.6)$ & 0.29 & .76 \\
\hline Ansiedad & $13.6(4.1)$ & $12.5(4.4)$ & 1.33 & .18 \\
\hline Depresión & $10.4(4.4)$ & $10.1(4.4)$ & 0.41 & .68 \\
\hline Función Física & $21.0(5.0)$ & $20.4(6.3)$ & 0.47 & .63 \\
\hline Impacto & $12.5(5.5)$ & $13.5(5.6)$ & -0.92 & .35 \\
\hline Síntomas & $35.8(9.0)$ & $36.1(9.2$ & -0.18 & .95 \\
\hline Impacto fibromialgia & $69.3(16.9)$ & $70.0(19)$ & -0.19 & .84 \\
\hline${ }^{\mathrm{a} e n}$ tabla: media (desv & ión estándar); ${ }^{+}$siq & ión marginal ${ }^{*}$ & $p<.01$ & \\
\hline
\end{tabular}

Tabla 3.- Correlaciones entre preferencia de metas y patrones de actividad

\begin{tabular}{lc}
\hline Patrones de actividad & $\begin{array}{c}\text { Preferencia de } \\
\text { metas }^{\mathrm{a}}\end{array}$ \\
\hline Evitar dolor & $.39^{* *}$ \\
Evitar actividad & $.27^{* *}$ \\
Persistencia contingente a la tarea & $-.35^{* *}$ \\
Persistencia excesiva & $-.24^{* *}$ \\
Persistencia contingente al dolor & -.02 \\
Regular la actividad para incrementarla & $.28^{* *}$ \\
Regular la actividad para conservar la energía & $.31^{* *}$ \\
Regular la actividad para reducir el dolor & $.39^{* *}$
\end{tabular}

${ }^{a}$ A mayor puntuación, mayor preferencia por evitar dolor;

${ }^{*} \mathrm{p}<.05 ;{ }^{* *} \mathrm{p}<.01$ 
trones de actividad salvo con la persistencia contingente al dolor (Tabla 3). Las correlaciones fueron de signo positivo en el caso de los patrones de evitación y de regulación de la actividad (pacing), oscilando entre $r=.39(p<.000)$ y $r=.27$ ( $p<.000)$, mientras que fueron de signo negativo en el caso de los patrones de persistencia, siendo la más alta la obtenida con el patrón de persistencia en la tarea $(r=-.35 ; p<.000$ ) (Tabla 3).

\section{Discusión}

La investigación de la psicología aplicada al deporte y a la actividad física se ha centrado en estudiar los procesos psicológicos implicados en la actividad deportiva, así como en los factores y estrategias que facilitan el rendimiento deportivo. Sin embargo, comparado con los estudios realizados en el ámbito del rendimiento e incluso de la iniciación, la producción científica dedicada a poblaciones específicas relacionadas con ejercicio y salud ha sido, aunque relevante, de menor cantidad. En la última década, han proliferado estudios de instrumentos de evaluación psicológica en el ámbito del deporte, pero son escasos los que se centran en la evaluación de factores psicológicos relacionados con ejercicio físico en poblaciones con problemas de salud crónicos.

El estudio presentado ha tenido un doble objetivo. En primer lugar, ha explorado la preferencia de metas relacionadas con el ejercicio físico en mujeres con fibromialgia, analizando si existe un perfil de pacientes que prefiera mantenerse realizando la actividad física en lugar de evitar el dolor. En este sentido, los datos obtenidos indican que no hay características sociodemográficas ni clínicas asociadas a la preferencia de metas en este contexto de ejercicio. De hecho, sólo aquellas mujeres que se ocupan del trabajo en casa muestran mayor preferencia por metas de ejercicio. Los resultados indican, por tanto, que no existe un perfil específico de mujeres con fibromialgia que prefieran metas hedónicas frente a metas de ejercicio físico. Sin embargo, aunque las diferencias entre ambos grupos no sean significativas, se observaron tendencias que merece la pena comentar. En este sentido, las mujeres más mayores prefieren metas evitadoras de dolor mientras que las más jóvenes prefieren metas relacionadas con el ejercicio. Además, aquellas mujeres con preferencia de metas de ejercicio físico muestran puntuaciones ligeramente más elevadas en ansiedad. Estos resultados podrían explicarse en función de la importancia que tienen las variables situacionales en relación con la preferencia de metas, posiblemente relacionadas con el valor que las mujeres le dan a la meta. Algunas teorías motivacionales, aplicadas al contexto deportivo, han resaltado la importancia del valor incentivo de la meta y de la interacción entre los factores personales y situacionales que pueden llegar a explicar la intensidad y dirección de la conducta (Atkinson y Raynor,1974; McClelland, Atkinson, Clark y Lowell, 1953).

Respecto al segundo objetivo, que consistió en mostrar las relaciones existentes entre la preferencia de metas y los patrones de actividad física, los datos muestran numerosas relaciones entre la preferencia de metas en el contexto del ejercicio físico y los patrones de actividad física en mujeres con fibromialgia. En este sentido, la preferencia metas por el ejercicio que muestran las mujeres y la persistencia ante él indica un patrón coherente de relaciones. Parece lógico que aquellas mujeres que prefieren realizar ejercicio persistan en la tarea en lugar de abandonarla. Por otra parte es coherente también que aquellas pa- 
cientes que prefieren evitar el dolor en el contexto del ejercicio físico, muestren patrones de actividad evitadores, suponiendo un futuro reto de investigación la profundización en las relaciones obtenidas entre la preferencia por la evitación del dolor vs ejercicio y los patrones de actividad relacionados con la regulación de la misma (pacing).

Sin duda, los enormes avances médicos y el envejecimiento de la población han transformado la enfermedad mortal en una patología crónica, convirtiéndose a día de hoy, en las responsables del $63 \%$ de las muertes a nivel mundial (OMS, 2019), lo que hace de la enfermedad crónica un tema de estudio científico en auge. En este contexto, el ejercicio físico ha sido recomendado por instituciones sanitarias en la prevención de enfermedades y el mantenimiento de la salud a lo largo del ciclo vital por los beneficios que reporta a nivel físico, psicológico y social (Ministerio de Sanidad, Consumo y Bienestar Social, 2018). Trabajar por y para el mantenimiento de la salud al través del ejercicio es sin duda relevante, teniendo en cuenta las particularidades las poblaciones con una enfermedad crónica. En poblaciones específicas como es la fibromialgia, se ha constatado la importancia de trabajar en este contexto de ejercicio por los inhibidores potenciales del mismo, como es el dolor o la fatiga (Pastor et al., 2015).

En futuros estudios sería de sumo interés abordar los aspectos psicológicos y la conducta de ejercicio aplicando intervenciones psicológicas específicas, tanto en fibromialgia como en pacientes de otros dolores crónicos, y siguiendo una metodología de estudio rigurosa que permita, por una parte, evaluar el efecto de la intervención y, por otra, diseñar protocolos de actuación en las pacientes para, finalmente, ampliar el conocimiento acerca de esta patología. En este sentido algunos trabajos previos han demostrado la eficacia a corto plazo de las intenciones de implementación en la conducta de caminar en pacientes de fibromialgia (Pastor et al., 2014; Pastor-Mira, López-Roig, Peñacoba, Martínez-Zaragoza, Sanz, León, ... Schweiger, 2017).

Finalmente, conviene incidir en la importancia del establecimiento de metas como recurso de trabajo ya que esta técnica ha demostrado su eficacia en el contexto del ejercicio físico, por ejemplo, en deportistas de alto rendimiento (Ucha y Perez, 1988; Weinberg, 2013). Es necesario que los y las profesionales de la psicología faciliten a las pacientes estrategias psicológicas que les permitan conseguir sus objetivos de la manera más eficaz posible. El equipo multidisciplinar que trabaja con mujeres afectadas por fibromialgia debería, por tanto, conocer en profundidad los factores y los efectos psicológicos derivados de la enfermedad y que afectan gravemente a la salud psicológica de sus pacientes. Por todo ello, las intervenciones tendrían que incidir en convertir el ejercicio en una meta valiosa para estas pacientes. Tal vez si se consigue este objetivo mejore la adhesión al ejercicio y el bienestar de las pacientes.

\section{Agradecimientos}

Los autores agradecen a las mujeres con fibromialgia que aceptaron participar en el estudio, así como a los profesionales de la Unidad de Fibromialgia de la Comunidad Valenciana (Hospital San Vicente del Raspeig), principalmente el Dr. Francisco Ortín y la Dra. Ester Abad, por su apoyo en todo el proceso de investigación. Los autores también agradecen a Esther Fernández-Díez su contribución en la recopilación e introducción de datos. 


\section{Proyecto de investigación subvencionado por el Ministerio de Economía, Industria y Com- petitividad (MINECO PSI2016-79566-C2-1-R).}

\section{Referencias}

Asociación Médica Mundial (1964). Declaración de Helsinki de la Asociación Médica Mundial. Principios éticos para las investigaciones médicas en seres humanos. (64a Asamblea General, Fortaleza, 2013). Recuperado de http:// www.wma.net/es/ 30publications/10policies/b3/

Atkinson, J. W., y Raynor, J. Q. (1974). Personality, motivation, and achievement. New York: Hemisphere.

Busch, A., Webber, S. C., Richards, R. S., Bidonde, J., Schachter, C. L., Schafer, L. A.,... Overend, T. J. (2013). Resistance exercise training for fibromyalgia. The Cochrane Database of Systematic Reviews, 12: CD010884.

Cantón, E. (2009). La profesión de Psicólogo del Deporte. Revista de Psicología General y Aplicada, 62(1-2), 121-129.

Craig, C. L., Marshall, A. L., Sjöström, M., Bauman, A. E., Booth, M. L., Ainsworth, B. E.,..., Oja, P. (2003). International Physical Activity Questionnaire: 12-Country Reliability and Validity. Official Journal of the American College of Sports Medicine, 35(8), 1381-95.

Christiansen, S., Oettingen, G., Dahme, B., y Klinger, R. (2010). A short goal-pursuit intervention to improve physical capacity: a randomized clinical trial in chronic back pain patients. Pain, 149, 444-452.

Karsdorp, P. A., y Vlaeyen, J. W. (2011). Goals matter: both achievement and pain-avoidance goals are associated with pain severity and disability in patients with low back and upper extremity pain. Pain, 152(6), 1382-1390.

Karsdorp, P.A., Geenen, R., Kroese, F.M. y Vlaeyen, J.W.S. (2016). Turning pain into cues for goal-directed behavior: Implementation intentions reduce escape-avoidance behavior on a painful task. Journal of Pain, 17(4), 499-507. doi:https://doi.org/10.1016/j.jpain.2015.12.014

Esteve-López, F., Pulido-Martos, M., Armitage, Ch., Wearden, A., Álvarez-Gallardo, I., Arrayás-Grajera, M.J.,...SeguraJiménez, V. (2016). Factor structure of the Positive and Negative Affect Schedule (PANAS) in adult women with fibromyalgia from Southern Spain: the al-Andalus project. PeerJ, 4, e1822. doi: https://doi.org/10.7717/ peerj.1822

Esteve, R., Ramirez-Maestre, C., Peters, M. L., Serrano-Ibanez, E. R., Ruiz-Parraga, G. T., \& Lopez-Martinez, A. E. (2016). Development and initial validation of the activity patterns scale in patients with chronic pain. The Journal of Pain, 17(4), 451-461.

Fernández- Cabrera, T., Medina- Anzano, S., Herrera-Sánchez, I., Rueda-Méndez, S., \& Fernández Del Olmo, A. (2011). Construcción y validación de una escala de autoeficacia para la actividad física. Revista Española de Salud Pública, 85(4), 405-417. Recuperado en 22 de mayo de 2019, de http://scielo.isciii.es/scielo.php?script=sci_ arttext\&pid=S1135-57272011000400009\&lng=es\&tlng=es.

Heckhausen, H. y Gollwitzer, P.M. (1987). Thought contents and cognitive functioning in motivational versus volitional states of mind. Motivation and Emotion, 11, 101-120.

León, E. y Cantón, E. (2008). La formación del profesional de la psicología del deporte. En J. A. Mora y F. Chapado (Eds.): Visión actual de la psicología del deporte (pp. 654-660). Sevilla: Editorial Wanceulen.

León, E. y Boix, S. (2018). Ejercicio físico y enfermedad crónica: Una relación beneficiosa para la salud. Boletín Informativo de la Federación Española de Psicología del Deporte, 26, 27-31.

López-Roig, S., Pastor, M. A., Peñacoba, C., Lledó, A., Sanz, Y. y Velasco, L. (2016). Prevalence and predictors of unsupervised walking and physical activity in a community population of women with fibromyalgia. Rheumatology International, 36, 1127-1133. doi:https://doi.org/10.1007/s00296-016-3508-1

McClelland, D. C., Atkinson, J. W, Clark, R. W. y Lowell, E. L. (1953). The achievement motive. New York: Appleton- Century Crofts.

Macfarlane, G. J., Kronisch, C., Dean, L. E., Atzeni, F., Häuser, W., Fluß, E.,... Jones, G. T. (2017). EULAR revised recommendations for the management of fibromyalgia. Annals of the Rheumatic Diseases, 76, 318-328. 
McLoughlin, M.J., Colbert, L.H., Stegner, A.J. y Cook, D.B. (2011). Are women with fibromyalgia less physically active than healthy women? Medical Science and Sports Exercise, 43, 905-912.

Ministerio de Sanidad, Consumo y Bienestar Social (2018). Principales problemas crónicos en España. Recuperado de http://www.mscbs.gob.es/estadEstudios/ sanidadDatos/home.htm

Ministerio de Sanidad, Consumo y Bienestar Social (2018). Actividad física y Salud en la infancia y Adolescencia: Guía para todas las personas que participen en su educación Recuperado de http://www.mscbs.gob.es/ciudadanos/proteccionSalud/adultos/actiFisica/docs/ActividadFisicaSaludEspanol.pdfhttp://www.mscbs.gob.es/ estadEstudios/ sanidadDatos/home.htm

Munguía-Izquierdo, D., Legaz-Arrese, A., y Mannerkorpi, K. (2011). Transcultural adaptation and psychometric properties of a Spanish-language version of physical activity instruments for patients with fibromyalgia. Archives of Physical Medicine and Rehabilitation, 92, 284-294.

Organización Mundial de la Salud (2019). Temas de salud. Enfermedades crónicas. Recuperado de https://www.who.int/ topics/chronic_diseases/es/

Organización Mundial de la Salud (2019). Ten threats to global health in 2019. Recuperado de https://www.who.int/emergencies/ten-threats-to-global-health-in-2019

Pastor, M.A., López-Roig, S., Lledó, A., Peñacoba, C., Velasco, L., Schweiger-Gallo, I.,...Sanz, Y. (2014). Combining motivational and volitional strategies to promote unsupervised walking in patients with fibromyalgia: study protocol for a randomized controlled trial. Trials, 15, 120. doi:10.1186/1745-6215-15-120

Pastor, M. A., López-Roig, S., Sanz, Y., Peñacoba, C., Cigarán, M., Lledó, A., et al. (2015). Andar como forma de ejercicio físico en la Fibromialgia: un estudio de identificación de creencias desde la Teoría de la Acción Planeada, Anales de Psicología, 31, 433-446. doi:https://doi.org/10.6018/analesps.31.2.172151

Pastor-Mira, M. A., López-Roig, S., Peñacoba, C., Martínez-Zaragoza, F., Sanz, Y., León, E., ... Schweiger, I. (2017). Using motivational and volitional interventions to promote unsupervised walking in women with fibromyalgia. The European Health Psychologist, 19(Supp.), 1075.

Kindermans, H.P.J., Roelofs, J., Goossens, M.E.J.B., Huijnen, I.P.J., Verbunt, J.A. y Vlaeyen, J.W.S. (2011). Activity patterns in chronic pain: underlying dimensions and associations with disability and depressed mood. The Journal of Pain: official journal of the American Pain Society, 12(10), 1049-1058.

Team, R. C. (2019). R: A language and environment for statistical computing. Vienna, Austria: R Foundation for Statistical Computing.

Sañudo, B., Galiano, D., Carrasco, L., \& de Hoyo, M. (2010). Evidencias para la prescripción de ejercicio físico en pacientes con fibromialgia. Revista Andaluza de Medicina del Deporte, 3(4), 159-169.

Salgueiro, M., García-Leiva, J.M., Ballesteros, J., Hidalgo, J., Molina, R. y Calandre, E. (2013). Validation of a Spanish version of the Revised Fibromyalgia Impact Questionnaire (FIQR). Health and Quality of Life Outcomes, 11, 132.

Terol, M.C., López-Roig, S., Rodríguez-Marín, J., Martín-Aragón, M., Pastor, M. A. y Reig, M.T. (2007). Propiedades psicométricas de la Escala Hospitalaria de Ansiedad y Depresión (HAD) en población española. Ansiedad y Estrés, 13, 163-176.

Ucha, E. y Perez, R. (1988). Establecimiento de metas: un procedimiento para incrementar los rendimientos deportivos. Educación Física y Deporte, 10(1-2), 23-36.

Van Damme, S., y Kindermans. H. (2015). A self-regulation perspective on avoidance and persistence behavior in chronic pain. New theories, new challenges? Clinical Journal of Pain, 31, 115-122

Vallejo, M. A., Rivera, J., Esteve-Vives, J. y Rodríguez-Muñoz, M. F. (2012). Uso del cuestionario Hospital Anxiety and Depression Scale (HADS) para evaluar la ansiedad y la depresión en pacientes con fibromialgia. Revista de Psiquiatría y Salud Mental, 5, 107-114.

Vlaeyen, J.W.S. y Linton, S.J. (2012). Fear-avoidance model of chronic musculoskeletal pain: 12 years on. Pain, 153, 11441147.

Weinberg, R. (2013). Goal setting in sport and exercise: research and practical applications. Revista Educación Física/ UEM, 24(2), 171-179. https://doi.org/10.4025/reveducfis.v24.2.17524 